\title{
Modelos de regressão aleatória para descrição da curva de crescimento de codornas de corte ${ }^{1}$
}

\author{
Cristina Moreira Bonafé2, Robledo de Almeida Torres ${ }^{3}$, José Lindenberg Rocha Sarmento ${ }^{4}$, \\ Luciano Pinheiro da Silva ${ }^{3}$, Jeferson Corrêa Ribeiro ${ }^{2}$, Rafael Bastos Teixeira ${ }^{3}$, Felipe Gomes \\ da Silva ${ }^{2}$, Mariele Freitas Sousa ${ }^{3}$
}

\footnotetext{
1 Fonte financiadora: Fapemig.

2 Departamento de Genética e Melhoramento/UFV, 36571-000, Viçosa - MG.

${ }^{3}$ Departamento de Zootecnia/UFV, 36571-000, Viçosa - MG.

${ }^{4}$ Departamento de Zootecnia/UFPI, 64900-000 - Bom Jesus - PI.
}

RESUMO - Objetivou-se com este trabalho comparar diferentes modelos de regressão aleatória, ajustados por meio de funções polinomiais de Legendre de diferentes ordens, para avaliar o que melhor se ajusta ao estudo genético da curva de crescimento de codornas de corte. Os dados utilizados são provenientes de 26.835 e 27.447 observações, de 3.909 e 4.040 codornas de corte das linhagens UFV-1 e UFV-2. O peso corporal nas duas linhagens foi avaliado aos 1, 7, 14, 21, 28, 35 e 42 dias de idade. Ordens de ajuste das funções contínuas foram gradualmente aumentadas (ordens variando de 3 a 6) para determinação da ordem mínima necessária para descrever as estruturas de covariância em função do tempo nos modelos de regressão aleatória. A função polinomial de Legendre com as ordens 6 para efeito genético aditivo direto e 5 para efeito permanente de animal, para a linhagem UFV-1, e 6 para ambos efeitos aleatórios, para a linhagem UFV-2, deve ser utilizada na avaliação genética da curva de crescimento de codornas de corte em estudo. As herdabilidades estimadas indicam que ganhos genéticos podem ser obtidos como resposta à seleção para peso corporal nas duas linhagens.

Palavras-chave: componente de variância, herdabilidade, polinômio de Legendre

\section{Random regression models for description of growth curve of meat quails}

\begin{abstract}
The objective of this paper was to compare different random regression models adjusted through Legendre polynomial functions of different orders to evaluate the one best adjusted to the genetic study of the meat quail growth curve. The data used in this study were from 26,835 and 27,447 observations from 3,909 and 4,040 meat quails of the UFV-1 and UFV-2 strain, respectively. The body weight of the two strain was evaluated on 1, 7, 14, 21, 28, 35 and 42 days of age. The orders of adjustment of the continuous functions were gradually increased (orders varying from 3 to 6), to determine the least necessary order to describe structure of covariance in function of time in the random regression models. Legendre polynomial functions of the $6^{\text {th }}$ order for direct additive genetic effect and $5^{\text {th }}$ order for permanent effect, for strain UFV-1 and 6 for both random effects for strain UFV-2 should be used in the genetic evaluation of the quail meat growth curve in study. The estimated heritability indicates which genetic traits can be obtained as a response to the physical body weight selection in two strain.
\end{abstract}

Key Words: heritability, Legendre polynomial, variance component

\section{Introdução}

Regressões polinomiais têm sido amplamente utilizadas para descrever a curva de crescimento dos animais. A maioria dos autores utiliza regressões sobre polinômios de Legendre para modelar dados longitudinais. Estes modelos não requerem qualquer pressuposição sobre a forma da curva ou trajetória da característica com o tempo. Entretanto, em geral, requerem o ajuste de polinômios de alto grau, especialmente para modelar os efeitos de ambiente permanente de animal (Meyer, 1999; Brotherstone et al., 2000; Meyer, 2000; Meyer, 2001) e, em consequência, apresentam um grande número de parâmetros a ser estimado. Polinômios de alto grau são associados a problemas numéricos (Kirkpatrick et al., 1994; Meyer, 1998b) e a grandes exigências computacionais.

Assim, torna-se necessária a utilização de métodos que consigam agrupar toda a informação de um animal, estimar mais precisamente os fatores de ambiente com a utilização dos dados observados, visando incrementar a acurácia, as 
possibilidades de ganhos genéticos e o melhoramento genético. Ao ajustar um modelo de regressão aleatória, implicitamente, ajusta-se uma função contínua que permite descrever as mudanças genéticas e ambientais com o passar do tempo. Vários trabalhos têm empregado como funções contínuas os polinômios ortogonais de Legendre, os quais podem adequar a ordens de ajustes diferentes para cada efeito aleatório contido no modelo de análise. A falta de um modelo apropriado, isto é, o desconhecimento da ordem de ajuste mais apropriada para a função empregada, pode influenciar erroneamente a partição da variância fenotípica nas variâncias atribuídas aos efeitos incluídos no modelo (Sarmento, 2007).

Dessa forma, objetivou-se com este trabalho comparar diferentes modelos de regressão aleatória por meio de funções polinomiais de Legendre de diferentes ordens, para avaliar o que melhor se ajusta ao estudo genético da curva de crescimento de codornas de corte.

\section{Material e Métodos}

Os dados utilizados neste estudo são provenientes de duas linhagens experimentais distintas de codornas de corte, pertencentes ao Programa de Melhoramento Genético de Aves da Universidade Federal de Viçosa. Foram utilizados 26.835 registros de pesos para a linhagem UFV-1 e 27.447 para a UFV-2. Os pesos foram mensurados a cada sete dias, ou seja, ao nascimento, 7, 14, 21, 28, 35 e 42 dias de idade. Os dados foram provenientes de 3.909 e 4.040 codornas de corte, com 6.742 e 7.381 animais na matriz de parentesco, das linhagens UFV-1 e UFV-2, respectivamente.

Os pesos corporais foram analisados por meio de modelo animal em regressão aleatória. As regressões fixas e aleatórias foram representadas por funções contínuas, cujas idades foram descritas em termos de polinômios ortogonais de Legendre, as ordens de ajuste das funções contínuas foram gradualmente aumentadas (ordens variando de 3 a 6 ), para determinar a ordem mínima necessária para descrever as estruturas de covariâncias em função do tempo nos modelos de regressão aleatória. A variância residual foi considerada heterogênea por meio de sete classes de idades $(1,7,14,21$, 28, 35 e 42 dias).

Polinômios ortogonais de Legendre da idade foram utilizados como funções contínuas para representar as regressões: fixa e aleatória. Generalizando, os modelos ajustados por funções de diferentes ordens para os efeitos aleatórios podem ser representados como segue:

$$
y_{i j}=F+\sum_{m=1}^{3} \beta_{m} \phi_{m}+\sum_{m=1}^{k_{a}} \alpha_{i m} \phi_{m}+\sum_{m=1}^{k_{c}} \rho_{i m} \phi_{m}+\varepsilon_{i j}
$$

em que: $y_{i j}=$ peso no dia $j$ da codorna $i ; F=$ efeito fixo do grupo de contemporâneo (geração-eclosão-sexo-idade, com 126 classes); $\beta_{m}=$ coeficiente de regressão fixo do peso sobre o polinômio de Legendre $m$, representado por uma função quadrática, para modelar a curva média de crescimento da população; $\alpha_{i m}$ e $\rho_{i m}=$ coeficientes de regressão genético aditivo direto e de ambiente permanente do animal, respectivamente, para a codorna $i ; k_{a}$ e $k_{c}=$ ordens de ajustes dos polinômios de Legendre correspondentes, as quais variaram de 1 a $6 ; \phi_{m}=$ função polinomial de Legendre da idade padronizada $m$; e $\varepsilon_{i j}=$ efeito aleatório residual.

$\mathrm{Na}$ forma matricial, o modelo anterior, com suas respectivas pressuposições, pode ser escrito como: $y=X b+Z_{1} a+Z_{2} c+e$

$E\left[\begin{array}{c}y \\ a \\ c \\ e\end{array}\right]=\left[\begin{array}{c}X b \\ 0 \\ 0 \\ 0\end{array}\right] \quad \begin{array}{r}\operatorname{Var}(a)=K_{a} \otimes A, \\ \operatorname{Var}(c)=K_{c} \otimes I_{N d},\end{array}$

em que $y=$ vetor de $N$ observações referentes a $N_{d}$ animais; $b=$ vetor que contém os efeitos fixos e os coeficientes $b_{m}$ da regressão fixa; $a=$ vetor $k_{a} \times N_{D}$ de coeficientes de regressão aleatória genéticos aditivos diretos, em que $N_{D}$ representa o número de animais na matriz de parentesco; $c=$ vetor $k_{c} \times N_{d}$ de coeficientes de regressão aleatória de ambiente permanente de animal; $e$ é um vetor de erros aleatórios; $X, Z_{1} \mathrm{eZ}_{2}=$ matrizes de incidência dos coeficientes de regressão fixos, coeficientes de regressão aleatória genético aditivo direto e ambiente permanente de animal, respectivamente. $K_{a}$ e $K_{c}=$ matrizes de covariâncias entre os coeficientes de regressão aleatórios genético aditivo direto e ambiente permanente de animal, respectivamente; $A=$ matriz dos numeradores do coeficiente de parentesco entre os indivíduos; $I_{N d}=$ matriz identidade de dimensão $N_{d}$; $\otimes=$ operador produto direto; $\sigma_{j}^{2}=$ variâncias residuais.

Para permitir um estudo mais detalhado, foi utilizado um modelo que corresponde a um modelo de repetibilidade, com $\mathrm{k}_{\mathrm{a}}=1 \mathrm{e}_{\mathrm{c}}=1$, equivalente a $\mathrm{k}=11$, em que apenas o intercepto foi ajustado.

As ordens de ajuste das funções contínuas foram aumentadas gradativamente, para se determinar a ordem mínima necessária para descrever as variâncias e a estrutura de covariância em função do tempo para cada efeito aleatório.

Os diferentes modelos foram comparados pelo teste da razão de verossimilhança (LRT), critério de informação de Akaike (AIC) e o critério de informação Bayesiano de Schwarz (BIC).

Além disso, os componentes de covariâncias e parâmetros genéticos estimados ao longo da curva de 
crescimento obtidos pelos modelos que empregaram as funções com as melhores ordens de ajuste foram inspecionados e comparados aos estimados por meio de análise unicaracterística usando modelo animal.

As covariâncias entre os coeficientes de regressão aleatória genéticos aditivos direto e ambiente permanente de animal, conforme o modelo ajustado, foram estimadas pelo método da máxima verossimilhança restrita (REML), usando o programa DXMRR do software DFREML (Meyer, 1998a).

\section{Resultados e Discussão}

O emprego de modelos de regressão aleatória por meio de funções polinomiais de Legendre requer a definição da ordem mais apropriada para cada efeito aleatório considerado no modelo de análise, assim, as ordens de ajuste das funções contínuas foram gradualmente aumentadas (ordens variando de 1 a 6), visando determinar a ordem mínima necessária para descrever as estruturas de covariâncias em função do tempo.

O modelo de repetibilidade $(\mathrm{k}=11)$ apresentou os piores valores para todos os critérios utilizados (Tabela 1 ), o que indica que as estimativas de variâncias constantes não são apropriadas para o estudo apresentado. Resultados semelhantes foram obtidos por Meyer (1998b) e Sakaguti (2000) em estudos com bovinos de corte, e por Sarmento (2007), em ovinos de corte.

Os ajustes linear $(\mathrm{k}=22)$ e quadrático $(\mathrm{k}=33)$ propiciaram valores maiores para o $\log _{\mathrm{e}} \mathrm{L}$ ( $\mathrm{P}<0,01$ pelo LRT) e menores de AIC e BIC, o que indica que estes apresentam melhores ajustes em relação aos modelos que as variâncias foram mantidas constantes, mas foram piores que os valores obtidos com modelos de maiores ordens de ajustes, que sugere que esses modelos propiciaram ajustes simplificados para representar as mudanças nas variâncias em função do tempo.

De acordo com $\log _{e} L$, as ordens mais altas dos polinômios para os efeitos produziram maiores valores, o que sugere que o modelo mais parametrizado (modelo 66, com 49 parâmetros a serem estimados) com $\mathrm{k}_{\mathrm{a}}=6$ para os efeitos genético aditivo direto $\mathrm{k}_{\mathrm{c}}=6$ para ambiente permanente de animal, proporcionou melhor ajuste para as duas linhagens. Entretanto, de acordo com o resultado do teste da razão de verossimilhança (LRT), este modelo não diferiu estatisticamente $(\mathrm{P}>0,01)$ do modelo 65 para a linhagem UFV-1, o que indica que maiores ordens não proporcionam melhorias para ajuste do efeito permanente de ambiente.

Os modelos com melhores ajustes foram: o 65 para a linhagem UFV-1 e o 66 para a linhagem UFV-2 (Tabela 1), com base nos resultados observados pelo Critério de Informação Bayesiano (BIC) e o Critério de Informação de Akaike(AIC).

Akbas et al. (2004) verificaram melhor ajuste quando admitiram ordem 6 para os efeitos aleatórios e ao considerarem variância residual constante para todas as idades.

O intercepto explicou a maior proporção da variância para os efeitos aleatórios incluídos nos dois modelos (Tabela 2) para ambas as linhagens. Quando empregado o modelo mais parametrizado, houve maior número de

Tabela 1 - Teste para comparação dos modelos

\begin{tabular}{|c|c|c|c|c|c|c|}
\hline & Modelo $^{1}$ & NP & $\log _{e} L^{2}$ & $\mathrm{AIC}^{2}$ & $\mathrm{BIC}^{2}$ & L RT \\
\hline \multirow[t]{10}{*}{ UFV-1 } & 11 & 9 & $-11113,6$ & 22147,4 & 21868,7 & - \\
\hline & 22 & 13 & $-3473,1$ & 6874,4 & 6628,5 & $7640,5^{* *}$ \\
\hline & 33 & 19 & $-1620,9$ & 3182,0 & 2985,3 & $1852,2^{* *}$ \\
\hline & 43 & 23 & $-921,2$ & 1790,7 & 1626,7 & $699,4^{* *}$ \\
\hline & 44 & 27 & $-664,1$ & 1284,3 & 1153,1 & $257,5^{* *}$ \\
\hline & 53 & 28 & $-596,1$ & 1150,4 & 1027,4 & $67,9^{* *}$ \\
\hline & 54 & 32 & $-336,0$ & 638,2 & 548,0 & $56,4^{* *}$ \\
\hline & 64 & 38 & $-307,6$ & 593,4 & 552,4 & $164,0^{* *}$ \\
\hline & 65 & 43 & $-5,9$ & 0 & 0 & $301,7^{* *}$ \\
\hline & 66 & 49 & 0 & 0,2 & 49,4 & 5,9ns \\
\hline \multirow[t]{10}{*}{ UFV-2 } & 11 & 9 & $-20537,6$ & 40995, 2 & 40666,4 & - \\
\hline & 22 & 13 & $-12806,4$ & 25540,7 & 25244,8 & $7731,2^{* *}$ \\
\hline & 33 & 19 & $-10951,8$ & 21843,6 & 21597,0 & $1854,6^{* *}$ \\
\hline & 43 & 23 & $-10362,5$ & 20672,9 & 20459,2 & $589,3^{* *}$ \\
\hline & 44 & 27 & $-10152,6$ & 20261,2 & 20080,4 & $209,9^{* *}$ \\
\hline & 53 & 28 & $-10057,6$ & 20073,3 & 19900,6 & $95,0^{* *}$ \\
\hline & 54 & 32 & $-9913,8$ & 19793,5 & 19653,8 & $143,9^{* *}$ \\
\hline & 64 & 38 & $-9807,4$ & 19592,8 & 19502,3 & $48,5^{* *}$ \\
\hline & 65 & 43 & $-9476,4$ & 18940,9 & 18891,5 & $331,0^{* *}$ \\
\hline & 66 & 49 & 0 & 0 & 0 & $13104,1^{* *}$ \\
\hline
\end{tabular}


autovalores associado à matriz dos coeficientes próximos de zero, o que pode ter sido causado pelo aumento da multicolineariadade, decorrente do aumento da ordem de ajuste da função empregada, o que sugere redução na dimensionalidade. Resultado similar, embora ligeiramente menor, foi encontrado por Akbas et al. (2004).

Modelos mais parcimoniosos devem ser preferidos, pois, de acordo com Legarra et al. (2004), ajuste perfeito aumenta a demanda computacional (memória e capacidade de processamento) e a susceptibilidade a erros numéricos. Os autores supracitados alertam para o fato de que a redução da dimensionalidade devido à eliminação de autovalores próximos de zero não é indicada em todos os casos, uma vez que, ao adotar este critério, pode resultar em modelagem simplista ou inadequada. Todavia, a seleção da ordem do polinômio a ser utilizada não é uma tarefa fácil e, para isso, os autovalores podem ser uma ferramenta útil (Foulley \& Robert-Granié, 2002). Assim, optou-se por inspecionar os componentes de variância estimados pelos diferentes modelos e os parâmetros genéticos resultantes apresentando-se os resultados dos dois modelos que melhor ajustaram aos dados.

Sarmento et al. (2008), comparando modelos de regressão aleatória para caprinos leiteiros, observaram que os valores do logaritmo da função de verossimilhança, de
AIC e BIC apontaram para seleção de modelos com ordens mais altas, entretanto, os autovalores associados às matrizes de covariâncias entre os coeficientes de regressão indicaram a possibilidade de redução da dimensionalidade. As altas ordens de ajuste proporcionaram estimativas de variâncias genéticas, correlações genéticas e de ambiente permanente não condizentes com o fenômeno biológico estudado, sendo um modelo mais parcimonioso suficiente para ajustar as variâncias nos dados

As estimativas das variâncias genéticas aditivas, de ambiente permanente de animal e fenotípicas obtidas pelos modelos 65 e 66 para a linhagem UFV-1 foram muito semelhantes (Figura 1), o que confirma a possibilidade de se usar o modelo 65 por ser mais parcimonioso e não causar grandes alterações nas estimativas dos componentes de variância.

A variância genética aditiva estimada por ambos os modelos aumentou do nascimento aos 42 dias de idade, e tornou-se ligeiramente superior a partir dos 35 dias para o modelo 66 da linhagem UFV-2. Estimativas de variâncias genéticas aditivas crescentes do nascimento aos 42 dias de idade, para codornas de corte, também foram relatadas por Akbas et al. (2004) e Dionello et al. (2006).

Estimativas de variâncias genéticas aditivas diretas crescentes ao longo da trajetória foram relatadas em ovinos

Tabela 2 - Estimativas de variâncias (diagonal), covariâncias (abaixo da diagonal) e correlações (acima da diagonal)

\begin{tabular}{|c|c|c|c|c|c|c|c|}
\hline & 1 & 2 & 3 & 4 & 5 & 6 & Autovalor (\%) \\
\hline \multirow[t]{13}{*}{ UFV-1 } & \multicolumn{7}{|c|}{ Efeito genético aditivo direto $\left(\mathrm{k}_{\mathrm{a}}=6\right)$} \\
\hline & 114,68 & 0,92 & $-0,32$ & $-0,66$ & 0,27 & 0,26 & 92,20 \\
\hline & 68,93 & 48,78 & 0,02 & $-0,70$ & 0,03 & 0,13 & 6,36 \\
\hline & $-8,73$ & 0,29 & 6,44 & 0,31 & $-0,77$ & $-0,49$ & 1,05 \\
\hline & $-10,06$ & $-6,94$ & 1,11 & 2,04 & $-0,21$ & $-0,55$ & 0,21 \\
\hline & 2,34 & 0,18 & $-1,57$ & $-0,24$ & 0,64 & 0,65 & 0,17 \\
\hline & 1,70 & 0,58 & $-0,77$ & $-0,49$ & 0,33 & 0,39 & 0,01 \\
\hline & \multicolumn{7}{|c|}{ Efeito de ambiente permanente de animal $\left(\mathrm{k}_{\mathrm{C}}=5\right)$} \\
\hline & 153,67 & 0,83 & $-0,54$ & $-0,35$ & 0,41 & & 86,23 \\
\hline & 74,78 & 52,65 & $-0,05$ & $-0,47$ & 0,12 & & 11,36 \\
\hline & $-28,34$ & $-1,50$ & 18,26 & 0,25 & $-0,65$ & & 1,79 \\
\hline & $-8,01$ & $-6,25$ & 1,92 & 3,33 & 0,24 & & 0,60 \\
\hline & 6,37 & 1,05 & $-3,43$ & 0,55 & 1,55 & & 0,01 \\
\hline \multirow[t]{14}{*}{ UFV-2 } & \multicolumn{7}{|c|}{ Efeito genético aditivo direto $\left(\mathrm{k}_{\mathrm{a}}=6\right)$} \\
\hline & 129,40 & 0,93 & 0,12 & $-0,70$ & 0,63 & 0,79 & 93,40 \\
\hline & 86,44 & 67,49 & 0,40 & $-0,77$ & 0,54 & 0,77 & 5,17 \\
\hline & 3,30 & 7,83 & 5,60 & $-0,07$ & $-0,38$ & 0,17 & 1,03 \\
\hline & $-11,24$ & $-8,88$ & $-0,23$ & 1,99 & $-0,53$ & $-0,84$ & 0,30 \\
\hline & 6,21 & 3,85 & $-0,78$ & $-0,65$ & 0,75 & 0,52 & 0,10 \\
\hline & 7,67 & 5,37 & 0,34 & $-1,01$ & 0,39 & 0,72 & 0,00 \\
\hline & \multicolumn{7}{|c|}{ Efeito de ambiente permanente de animal $\left(\mathrm{k}_{\mathrm{C}}=6\right)$} \\
\hline & 170,88 & 0,81 & $-0,53$ & $-0,47$ & 0,18 & $-0,05$ & 85,61 \\
\hline & 79,02 & 55,53 & $-0,02$ & $-0,66$ & $-0,17$ & $-0,30$ & 12,12 \\
\hline & $-30,36$ & $-0,75$ & 19,32 & 0,13 & $-0,86$ & $-0,43$ & 1,76 \\
\hline & $-8,98$ & $-7,15$ & 0,83 & 2,14 & $-0,26$ & $-0,40$ & 0,49 \\
\hline & 3,97 & $-2,17$ & $-6,36$ & $-0,63$ & 2,82 & 0,60 & 0,02 \\
\hline & $-0,62$ & $-2,34$ & $-1,97$ & $-0,61$ & 1,04 & 1,08 & 0,00 \\
\hline
\end{tabular}


de corte por Sarmento (2007), em bovinos de corte por Meyer (2001) e Albuquerque \& Meyer (2001), que também descreveram estimativas crescentes ao longo do período estudado, com ligeira tendência de diminuição ao final da curva de crescimento.

As variâncias estimadas para o efeito de ambiente permanente de animal e fenotípica (Figura 1) para a linhagem UFV-1 aumentaram à medida que o animal cresceu e praticamente não houve diferença nas trajetórias obtidas pelos dois modelos (65 e 66). Esses resultados indicaram que a inclusão dos coeficientes de regressão aleatória para esses efeitos permitiram diferenciar as variâncias estimadas ao longo da trajetória de crescimento, evidenciando que funções contínuas devem ser empregadas para modelá-los. Akbas et al. (2004), Dionello et al. (2006) e Winter (2005), encontraram a mesma tendência, em que a variância de ambiente permanente aumenta ao longo do tempo.

UFV-1

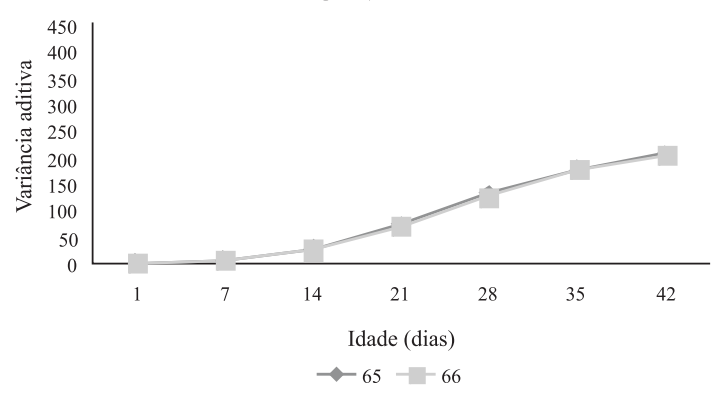

UFV-1

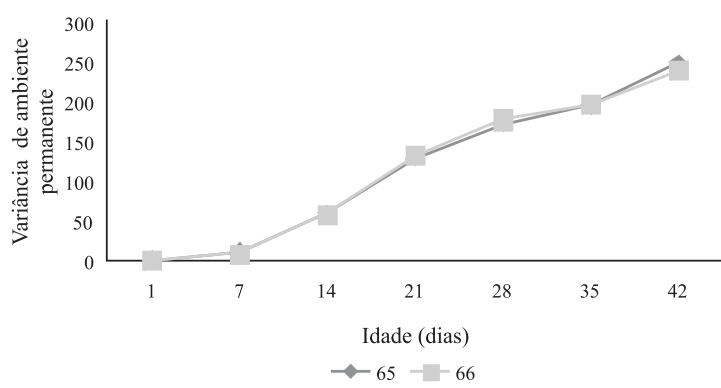

UFV-1

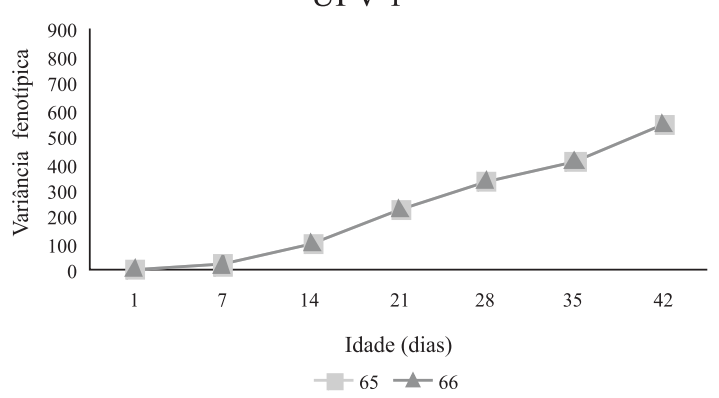

Dias et al. (2006), utilizando modelos de regressão aleatória para gado da raça Tabapuã observou aumento nas estimativas de variância para o efeito de ambiente permanente de animal em função da idade, sendo mais acentuado no final da curva. Tendências semelhantes foram relatadas por Albuquerque \& Meyer (2001) e Cyrillo (2003) para animais da raça Nelore.

As herdabilidades estimadas pelo ajuste dos modelos 65 e 66 para os dados da linhagem UFV-1, foram bem similares, reafirmando a possibilidade de se optar pelo modelo mais parcimonioso (65), diferente do comportamento dos mesmos modelos na linhagem UFV-2, que apresentaram variações maiores (Figura 2).

As herdabilidades estimadas foram de médias a altas, decrescentes do nascimento aos 14 dias, crescente dos 14 aos 35 dias e decrescendo novamente após os 35 dias. Dionello et al. (2008) encontraram herdabilidades crescentes para a

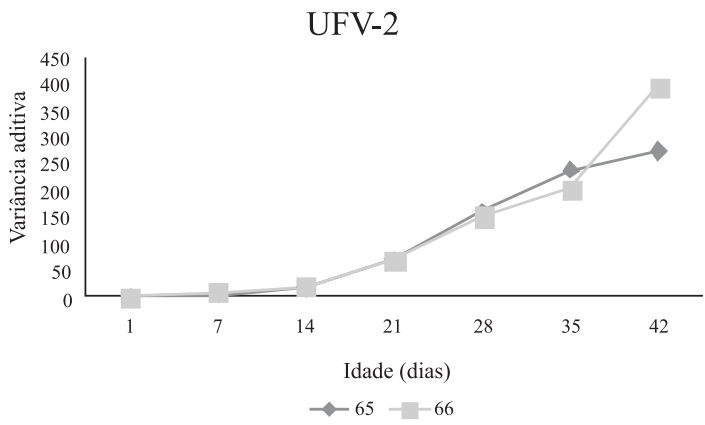

UFV-2

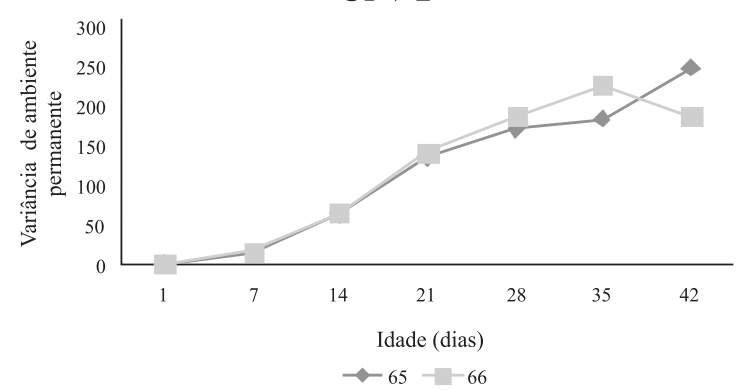

UFV-2

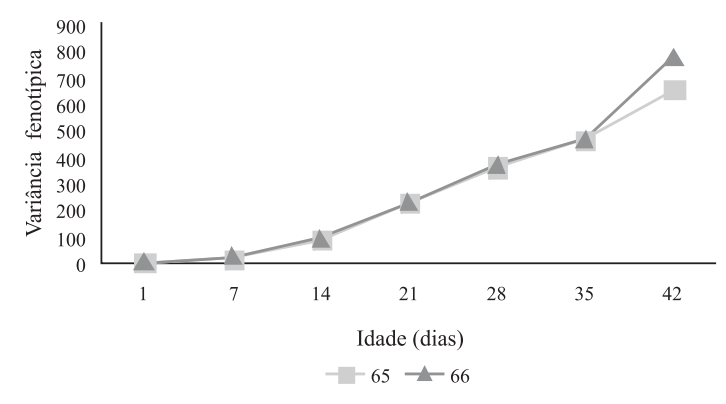

Figura 1 - Estimativas de componentes de variâncias genética aditiva, ambiente permanente de animal e fenotípica para os modelos 65 e 66. 
linhagem EV1, variando de 0,01 ao nascimento a 0,50 aos 42 dias de idade, porém, encontraram herdabilidades baixas, variando de 0,01 a 0,10 , para a linhagem EV2. Akbas et al. (2004) encontraram herdabilidades crescentes até 28 dias $(0,07$ a 0,61$)$ e depois leve queda até 42 dias de idade mantendo-se alta.

Para os dois modelos (66 e 65), as correlações entre peso nas diversas idades, à exceção entre peso ao nascer e os demais pesos, foram de média a altas e positivas (Tabela 3), assim como encontrados por Akbas et al. (2004) em estudo que considera modelo de ordem 6 e homogeneidade de variância residual.

As correlações de ambiente permanente estimadas entre os pesos das várias idades tenderam a ser maior entre idades mais próximas, entretanto, isso não foi verificado entre o peso ao nascer e os pesos obtidos dos 7 aos 14 dias, isto é, as correlações entre essas características foram muito baixas (Tabela 3).
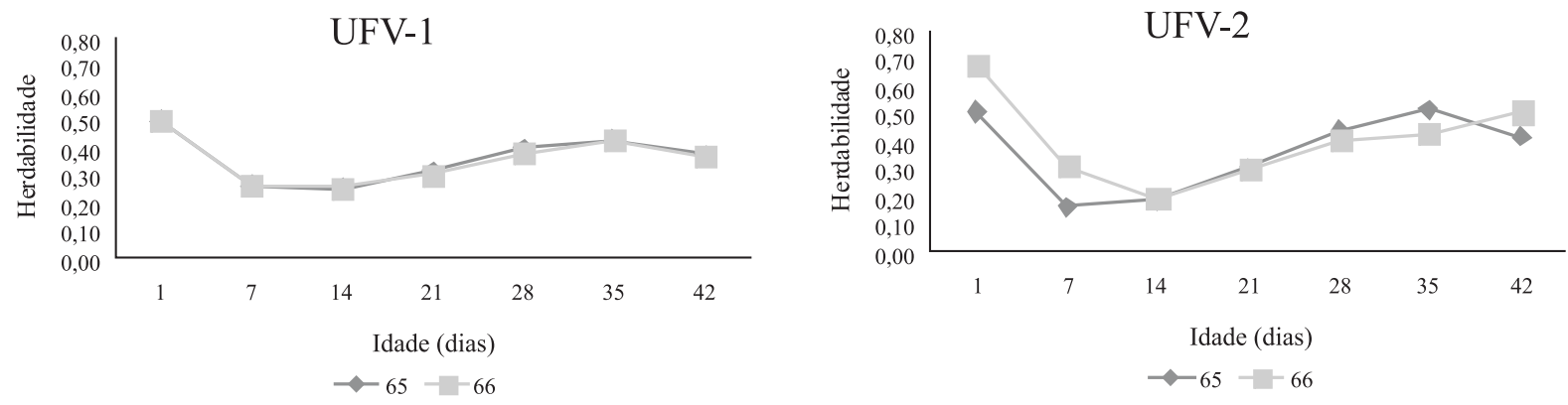

Figura 2 - Estimativas de herdabilidade para os modelos 65 e 66.

Tabela 3 - Estimativas das correlações genéticas (acima da diagonal) e de ambiente permanente (abaixo da diagonal) entre as idades estudadas para os modelos escolhidos para as duas linhagens

\begin{tabular}{|c|c|c|c|c|c|c|c|c|}
\hline \multicolumn{9}{|c|}{ 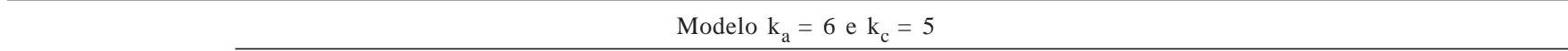 } \\
\hline UFV-1 & Idade & 1 & 7 & 14 & 21 & 28 & 35 & 42 \\
\hline & 1 & & 0,46 & 0,41 & 0,36 & 0,36 & 0,40 & 0,43 \\
\hline & 7 & 0,24 & & 0,85 & 0,69 & 0,56 & 0,44 & 0,41 \\
\hline & 14 & 0,15 & 0,83 & & 0,94 & 0,84 & 0,72 & 0,65 \\
\hline & 21 & 0,11 & 0,67 & 0,96 & & 0,97 & 0,86 & 0,79 \\
\hline & 28 & 0,09 & 0,53 & 0,84 & 0,95 & & 0,95 & 0,89 \\
\hline & 35 & 0,05 & 0,38 & 0,64 & 0,79 & 0,93 & & 0,97 \\
\hline & 42 & 0,02 & 0,27 & 0,48 & 0,60 & 0,73 & 0,88 & \\
\hline \multicolumn{9}{|c|}{ Modelo $\mathrm{k}_{\mathrm{a}}=6$ e $\mathrm{k}_{\mathrm{c}}=6$} \\
\hline UFV-2 & Idade & 1 & 7 & 14 & 21 & 28 & 35 & 42 \\
\hline & 1 & & 0,88 & 0,87 & 0,73 & 0,61 & 0,53 & 0,52 \\
\hline & 7 & 0,35 & & 0,79 & 0,54 & 0,42 & 0,35 & 0,31 \\
\hline & 14 & 0,29 & 0,80 & & 0,86 & 0,70 & 0,58 & 0,52 \\
\hline & 21 & 0,32 & 0,65 & 0,95 & & 0,96 & 0,88 & 0,83 \\
\hline & 28 & 0,33 & 0,56 & 0,85 & 0,95 & & 0,97 & 0,94 \\
\hline & 35 & 0,30 & 0,44 & 0,71 & 0,80 & 0,97 & & 0,99 \\
\hline & 42 & 0,31 & 0,35 & 0,59 & 0,71 & 0,88 & 0,97 & \\
\hline
\end{tabular}

\section{Conclusões}

Uma função polinomial de Legendre com as ordens 6, para efeito genético aditivo direto, e 5, para efeito permanente de animal, para a linhagem UFV-1 e com a ordem 6 para ambos os efeitos aleatórios para a linhagem UFV-2 deve ser utilizada na avaliação genética da curva de crescimento das codornas de corte em estudo.

\section{Agradecimentos}

À FAPEMIG, pelo financiamento do projeto. À CAPES e ao CNPq, pelas bolsas de estudos concedidas.

\section{Referências}

AKBAS, Y.; TAKMA, Ç.; YAYLAK, E. Genetic parameters for quail body weights using a randon regressino model. South 
African Jornal of Animal Science, v.34, n.2, p.104-109, 2004.

ALBUQUERQUE, L.G.; MEYER, K. Estimates of covariance functions for growth from birth to 630 days of age in Nelore cattle. Journal of Animal Science, v.79, p.2776-2789, 2001.

BROTHERSTONE, S.; WHITE, I.M.S.; MEYER, K. Genetic modelling of daily milk yield using orthogonal polynomials and parametric curves. Journal of Animal Science, v.70, p.417-416, 2000 .

CYRILLO, J.N.S.G. Estimativas de funções de covariância para o crescimento de machos Nelore utilizando modelos de regressão aleatória. 2003. 72f. Tese (Doutorado em Produção Animal) - Universidade Estadual Paulista, Jaboticabal.

DiONELLO, N.J.L.; SILVA, M.A.; CORREA, G.S.S. Genetic evaluation of European quail bt random regression analyses. In: WORLD CONGRESS ON GENETICS APPLIED TO LIVESTOCK PRODUCTION, 8., 2006, Belo Horizonte, MG, Brasil. Proceedings... Belo Horizonte, 2006.

DiONELLO, N.J.L.; CORREA, G.S.S.; SILVA. M.A. et al. Estimativas da trajetória genética do crescimento de codornas de corte utilizando modelos de regressão aleatória. Arquivo Brasileiro de Medicina Veterinária e Zootecnia, v.60, n.2, p.454-460, 2008.

KIRKPATRICK, M.; HILL, W.G.; THOMPSON, R. Estimating the covariance structure of traits during growth and aging, illustrated with lactation in dairy cattle. Genetics Research, v.64, n.57-69, 1994.

DIAS, L.T.; ALBUQUERQUE, L.G.; TONHATI, H. et al. Estimação de parâmetros genéticos para peso do nascimento aos 550 dias de idade para animais da raça Tabapuã utilizando-se modelos de regressão aleatória. Revista Brasileira de Zootecnia, v.35, n.5, p.1915-1925, 2006.

MEYER, K. “DXMRR” - A program to estimate covariance functions for longitudinal data by REML. In: WORLD
CONGRESS OF GENETICS APPLIED TO LIVESTOCK PRODUCTION, 6., 1998, Armidale. Proceedings... Armidale: University of New Wngland, 1998a. (CD ROM).

MEYER, K. Estimating covariance functions for longitudinal data using a random regression model. Genetics Selection Evolution, v.30, p.221-240, 1998b.

MEYER, K. Estimates of genetic and phenotypic covariance functions for postweaning growth and mature weight of beef cows. Journal of Animal Breeding and Genetics, v.116, p.181-205, 1999.

MEYER, K. Random regressions to model phenotypic variation in monthly weights of Australian beef cows. Livestock Production Science, v.65, p.19-38, 2000.

MEYER, K. Estimates of direct and maternal covariance functions for growth of Australian beef calves from birth to weaning. Genetics Selection Evolution, v.33, p.487-514, 2001.

SAKAGUTI, E.S. Funções de covariância e modelos de regressão aleatória na avaliação genética do crescimento de bovinos jovens da raça Tabapuã. 2000. 81f. Tese (Doutorado em Genética e Melhoramento) - Universidade Federal de Viçosa, Viçosa, MG.

SARMENTO, J.L.R. Modelos de regressão aleatória para avaliação genética da curva de crescimento de ovinos da raça Santa Inês. 2007. 101f. Tese (Doutorado em Genética e Melhoramento) - Universidade Federal de Viçosa, Viçosa, MG.

SARMENTO, J.L.R.; ALBUQUERQUE, L.G.; TORRES, R.A. et al. Comparação de modelos de regressão aleatória para estimação de parâmetros genéticos em caprinos leiteiros. Revista Brasileira de Zootecnia, v.37, n.10, p.1788-1796, 2008.

WINTER, E.M.W. Estimação de parâmetros genéticos de características de desempenho, carcaça e composição corporal de codornas para corte (Coturnix sp.). 2005. 91f. Dissertação (Mestrado em Genética - Melhoramento Animal) Universidade Federal do Paraná, Curitiba. 